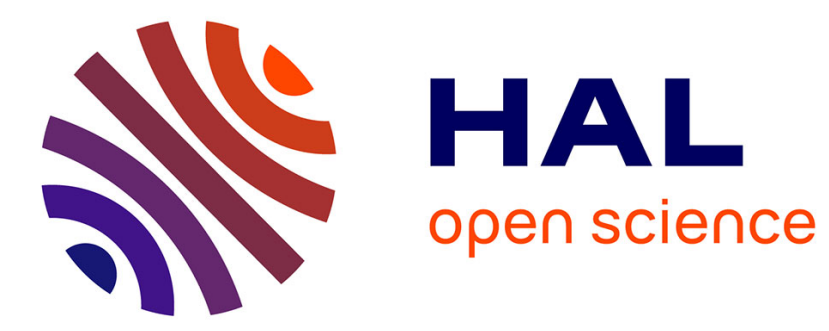

\title{
Di-nucleus dynamics towards fusion of heavy nuclei
}

\author{
Y. Abe, Caiwan Shen, G. Kosenko, D. Boilley, B.G. Giraud
}

\section{To cite this version:}

Y. Abe, Caiwan Shen, G. Kosenko, D. Boilley, B.G. Giraud. Di-nucleus dynamics towards fusion of heavy nuclei. International Journal of Modern Physics E, 2008, 17 (10), pp.2214-2220. 10.1142/S0218301308011367 . in2p3-00310894

\section{HAL Id: in2p3-00310894 https://hal.in2p3.fr/in2p3-00310894}

Submitted on 11 Aug 2008

HAL is a multi-disciplinary open access archive for the deposit and dissemination of scientific research documents, whether they are published or not. The documents may come from teaching and research institutions in France or abroad, or from public or private research centers.
L'archive ouverte pluridisciplinaire HAL, est destinée au dépôt et à la diffusion de documents scientifiques de niveau recherche, publiés ou non, émanant des établissements d'enseignement et de recherche français ou étrangers, des laboratoires publics ou privés. 


\title{
DI-NUCLEUS DYNAMICS TOWARD FUSION OF HEAVY NUCLEI
}

\author{
YASUHISA ABE \\ Research Center for Nuclear Physics, Osaka University, 10-1 Mihogaoka \\ Ibaraki, 567.0047/Osaka, Japan \\ abey@rcnp.osaka-u.ac.jp \\ CAIWAN SHEN \\ School of Science, Huzhou Tearchers College, \\ Huzhou, 313000/Zhejiang, P.R. China \\ cwshen@hutc.zj.cn \\ GRIGORY KOSENKO \\ Department of Physics, Omsk University, \\ Omsk, 644077, Russia \\ kosenko@phys.omsu.omskreg.ru \\ DAVID BOILLEY \\ GANIL, CEA/DSM-CNRS/INEP3, BP 55027, F-14076 Caen cedex 5, France \\ and Univ. of Caen, B.P. 5186, F-14032 Caen Cedex, France \\ boilley@ganil.fr \\ BERTRAND G. GIRAUD \\ Institut Physique Theorique, Direction des Sciences de la Matiere, Centre d'Etudes-Saclay, \\ Gif-sur-Yvette, F-91191, Fhance \\ bertrand.giraud@cea.fr \\ Received (received date) \\ Revised (revised date)
}

The Two-Step Model for fusion of massive systems is briefly recapitulated, which clarifies the mechanism of so-called fusion hindrance. Since the neck changes the potential landscape, especially the height of the conditional saddle point, time evolution of the neck degree of freedom plays a crucial role in fusion. We analytically solve time-evolution of nuclear shape of the composite system from di-nucleus to mono-nucleus. The timedependent distribution function of the neck is obtained, which elucidates dynamics of fusion processes in general, and thus, is useful for theoretical predictions on synthesis of the superheavy elements with various combinations of incident heavy ions.

\section{Introduction}

Theoretical prediction of productions of the superheavy elements (SHE) is one of the long-standing problems in nuclear physics. Based on the compound nucleus 
theory of nuclear reactions, cross sections are given by a product of the fusion and the survival probabilities. The latter is well-known, though there are ambiguities related to unknown parameters in the SHE region. But the mechanism of the fusion hindrance has not been clarified yet, although it has been experimentally known for a long time. Without its understanding, no reliable prediction is possible for cross sections of SHE. ${ }^{1}$ The present talk is mainly addressed to the problem, i.e., to the process from Di-Nucleus Configuration ( DNC ) formed by the incident combination of ions to the mono-nucleus, i.e., to the compound nucleus. For a description of nuclear shapes, we need at least three parameters. We employ the Two-Center parametrization, i.e., the distance $\mathrm{R}$, the asymmetry $\alpha$, and the neck parameter $\epsilon$. The parameter $\epsilon$ is not a neck radius, but defined by a modification factor of the spike in the di-harmonic potential. Nuclear shape is defined by an equipotential energy surface with a fixed volume. $\epsilon=1.0$ corresponds to no correction, thus to the configuration of touching two nuclei, while $\epsilon=0.0$ corresponds to no spike, thus to mono-nucleus with the neck being completely filled in. For the masssymmetric incident channels, the mono-nucleus is a compound nucleus with the superdeformation.

In the previous calculations, we simply assume $\epsilon$ to be equal to 0.8 , which has turned out to be not adequate as will be explained below. Due to that choice of the parameter, at least partially, we could not reproduce absolute peak values of the cross sections without adjustments of the shell correction energies in the calculations of the survival probabilities, ${ }^{2}$ by the refined statistical codes KEWPIE I and II. ${ }^{3}$

\section{Fusion Mechanism of Massive Systems ; Two-step model and the method of statistical connection}

A particular aspect in fusion of massive systems, which is different from that of lighter systems, is that the overcoming of the Coulomb barrier is not the end of the fusion process, as inferred from the hindrance observed experimentally. In fact, DNC formed by the incident combination of ions is located outside of the conditional saddle point, which is easily understood by the fact that DNC is a largely deformed compound system, while the saddle point for fission is located near the spherical ground state when the fissility parameter is close to 1 . Therefore, after overcoming the Coulomb barrier, the system has to overcome one more barrier, i.e., the conditional saddle point or the ridge-line. ${ }^{4}$

Therefore, the fusion probability $P_{\text {fusion }}$ is given by the product of the two probabilities: the probability for overcoming of the Coulomb barrier $P_{\text {sticking }}$ and that for the formation of the spherical compound nucleus, starting from DNC $P_{\text {form }}$.

$$
P_{\text {fusion }}\left(E_{c . m .}\right)=P_{\text {sticking }}\left(E_{c . m .}\right) \cdot P_{\text {form }}\left(E_{c . m .}\right) .
$$

An important point here is that DNC is already excited due to interactions before the contact of two ions, i.e., the incident kinetic energy is already dissipated. Therefore, the overcoming of the conditional saddle point is not made mechanically, 
but by the fluctuation. Actually, the calculations of passing over the Coulomb barrier are made by the use of the Surface Friction Model, extended to include the Langevin force associated with the friction. ${ }^{5}$ The results show that in all the incident systems for SHE, the incident kinetic energies are completely dissipated, i.e., are transferred into internal excitations. In this sense, the process is also a heatingup process of the compound systems. The radial momentum, thus, has a Boltzmann distribution with the corresponding temperature. This gives an initial condition for the next process of overcoming of the saddle point. That is, the two processes are connected statistically. The second process is solved by a multi-dimensional Langevin equation for shape evolution. For understanding of the two-step model with the statistical connection method, we show a schematic example of a onedimensional case with an inverted parabolic approximation for the barrier around the saddle point. ${ }^{6}$ The Langevin equation for the distance coordinate $q$ and the associate momentum $p$ is given as follows,

$$
\frac{d}{d t}\left[\begin{array}{l}
q \\
p
\end{array}\right]=\left[\begin{array}{cc}
0 & 1 / \mu \\
\mu \omega^{2} & -\beta
\end{array}\right] \cdot\left[\begin{array}{l}
q \\
p
\end{array}\right]+\left[\begin{array}{l}
0 \\
R
\end{array}\right],
$$

where $\mu, \omega$, and $\beta$ denote the inertia mass, the frequency of the parabola, and the reduced friction coefficient, respectively. $\beta=\gamma / \mu$ with the friction coefficient $\gamma$. The term $\mathrm{R}$ denotes the Langevin force, which is assumed to be Gaussian and related to the friction $\gamma$ by the dissipation-fluctuation theorem. Since the equation is linear, we can easily solve it. Then, we can obtain the probability for passing to the other side of the saddle, i.e., the formation probability with the initial values $q_{0}$ and $p_{0}$, by averaging over all the possible realizations of the random force $\mathrm{R}$ and by the integration over $p$ and the half $q$-space.

$$
\begin{aligned}
F_{\text {form }}\left(q_{0}, p_{0}, t\right) & =\int_{0}^{\infty} \frac{d q}{\sqrt{2 \pi}} \frac{1}{\sigma_{q}(t)} \exp \left(-\frac{(q-<q(t)>)^{2}}{2 \sigma_{q}^{2}(t)}\right) \\
& =\frac{1}{2} \operatorname{erfc}\left(-\frac{<q(t)>}{\sqrt{2} \sigma_{q}(t)}\right)
\end{aligned}
$$

where the average trajectory $\langle q(t)\rangle=A(t) \cdot q_{0}+B(t) \cdot p_{0}$. The coefficients $A(t)$, and $B(t)$ are given by the system constants: the friction $\gamma, \omega$ and $\mu$.

For a time long enough, the probability is written simply by an error function,

$$
\lim _{t \rightarrow \infty} F_{\text {form }}=\frac{1}{2} \operatorname{erfc}\left(\sqrt{\frac{x+\sqrt{x^{2}+1}}{2 x}} \sqrt{\frac{B}{T}}-\frac{1}{\sqrt{2 x\left(x+\sqrt{x^{2}+1}\right.}} \sqrt{\frac{K}{T}}\right),
$$

where $B=\mu \cdot \omega^{2} \cdot q_{0}^{2} / 2$, the saddle point height measured from the intial point $q_{0}$, while $K=p_{0}^{2} /(2 \cdot \mu) . x$ denotes the critical parameter $\beta /(2 \cdot \omega)$. As we discussed above, the distribution of $p_{0}$ is a Boltzmann type with a zero mean value, and then, an averaging overthe initial momentum $p_{0}$ gives an extremely simple expression for 
Y. Abe, C. Shen, G. Kosenko, D. Boilley and B.G. Giraud

the formation probability,

$$
P_{\text {form }}\left(E_{c . m .}\right)=\frac{1}{2} \operatorname{erfc}\left(\sqrt{\frac{B}{T}}\right) .
$$

Here, we can simply understand the reason why the fusion is hindered. Even if we give a larger incident kinetic energy, the formation and then the fusion probability does not increase accordingly. It increases very slowly through the increase of the temperature of the system, which appears in agreement with the experiment. ${ }^{7}$

\section{DNC to Mono-Nucleus; Filling-in of the neck cleft}

The fusion probability in the 1-dimensional model is further analysed, i.e., time evolutions of the formation probability and of the fusion flux are investigated, which are extremely helpful for an intuitive understanding of how the fusion process goes. In cases of low kinetic energies available at the contact configuration, the flux has a rather sharp peak in time around several in the unit of $\hbar / \mathrm{MeV}{ }^{8}$ Therefore, it is interesting to investigate how the other degrees of freedom affect the fusion process during the period of time. They couple with each other through Liquid Drop Model (LDM) potential energy, as well as through the inertia mass and the friction tensors, but the neck degree of freedom is relatively weak in the coupling with the others. Thus, it is meaningful to analyse time evolution of the neck degree of freedom, separately. As the first step, we look at the LDM potential for the symmetric incident systems, which turns out to be approximately linear in the neck parameter. ${ }^{9}$ We analyse time evolution of the neck parameter, starting at $\epsilon=1.0$ or around. A Langevin equation is solved, which turns out that the average value of the neck parameter changes very quickly, far quicker than the radial fusion for most systems including very heavy ones. ${ }^{10}$ The variance does also similarly. This is due to action of the linear driving force in the neck $\epsilon$, while the radial fusion is governed by the fluctuation or the diffusion. Thus, it suggests that the neck degree of freedom is in the thermal equilibrium during the fusion.

Next, in order to know how the distribution reaches the equilibrium, we try to obtain a time-dependent distribution function of the neck, starting from the deltafunction at $\epsilon=1.0$, i.e., at the initial DNC. The equation employed is Smoluchowski equation, since we know that the momentum space can be approximated to be in a Boltzmann distribution, due to a very small inertia mass and thus a very quick equilibration. ${ }^{11}$ With the linear potential, the equation to be solved is as follows,

$$
\frac{\partial N}{\partial t}=D \frac{\partial^{2} N}{\partial \epsilon^{2}}+C \frac{\partial N}{\partial \epsilon}
$$

where the diffusion coefficient $D=T / \gamma$, and $C=f / \gamma$ with the slope parameter $f$ : $V(\epsilon)=f \cdot \epsilon$. The friction coefficient $\gamma$ is calculated with the usual one body model, and the slope parameter $f$ is calculated with LDM. ${ }^{9}$ For simplicty, we take the range of the variable $\epsilon$ to be $[0.0, \infty]$, instead of the realistic $[0.0,1.0]$ (in this case, a little more complicated expression can be obtained). Nevertheless, this would be 
adequate enough, because of the linear potential which is unfavourable for larger $\epsilon$. The important boundary condition is that the point at $\epsilon=0.0$ is reflective. This is expressed in another way that the flux at $\epsilon=0.0$ is to be zero. As for the initial condition, the distribution at time $t=0$ is $\delta\left(\epsilon-\epsilon_{0}\right)$ with $\epsilon_{0}$ being a starting value of the neck parameter which should be close to 1.0, corresponding to the initial DNC. With the initial and the above boundary conditions, the solution is obtained as follows, ${ }^{12}$

$$
\begin{aligned}
N(\epsilon, t)= & \frac{1}{\sqrt{4 \pi D t}}\left[\exp \left(-\frac{\left(\epsilon-\epsilon_{0}\right)^{2}}{4 D t}\right)+\exp \left(-\frac{\left(\epsilon+\epsilon_{0}\right)^{2}}{4 D t}\right)\right] \\
& \times \exp \left(-\frac{C}{2 D}\left(\epsilon-\epsilon_{0}\right)-\frac{C^{2} t}{4 D}\right) \\
& +\frac{C}{2 D} \exp \left(-\frac{C \epsilon}{D}\right) \cdot \operatorname{erfc}\left(\frac{\epsilon+\epsilon_{0}-C t}{2 \sqrt{D t}}\right) .
\end{aligned}
$$

At the limit time $t$ goes to the infinity, or well later, the expression becomes to be a Boltzmann distribution. In Fig. 1, the time dependence is shown by distributions at several different times after the contact, for the case of ${ }^{100} \mathrm{Mo}+{ }^{100} \mathrm{Mo}$ system. Apparently, the Boltzmann distribution in the coordinate space is established al-

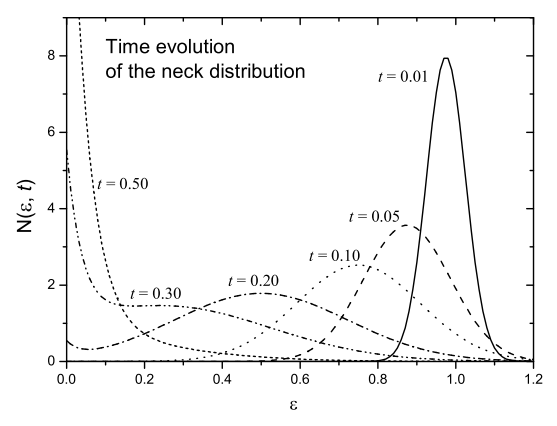

Fig. 1. Time evolution of the neck distribution function is shown with examples st several different times for ${ }^{100} \mathrm{Mo}+{ }^{100}$ Mo system, for which typical values of the parameters are $D=T / \gamma=1 / 8$ and $C=f / \gamma=20 / 8$ in the unit of $\mathrm{MeV} / \hbar$. The time unit is $\hbar / \mathrm{MeV}$.

ready at several tenths in the unit of $\hbar / \mathrm{MeV}$. It is worth mentioning that the time scale is far shorter than that of the 1-dimensional radial fusion. This means that before the radial motion for fusion starts, the neck cleft is filled in, i.e., the initial DNC becomes a superdeformed mono-nucleus. Thus, for fusioning motion, we can approximately take $\epsilon$ to be close to 0.0 , because it is the most probable value of the Boltzmann distribution obtained above.

\section{Remarks}

The time evolution toward the Boltzmann distribution from the initial delta function is not simple as seen in Fig. 1. Thus, for cases with the two time scales being 
not so different, we have to make a convolution over time in order to take into account effects of the neck degree of freedom, mainly changes of the saddle point height. Actually, the slope parameter $f$ is expected to depend on mass-asymmetry of DNC, because it is essentially due to the change in the surface area in the neck region. And the barrier height $\mathrm{B}$ is small in large mass asymmetries, then the two time scales would be not very different. Therefore, the time dependent distribution of the neck parameter presently obtained is important in understanding of the fusion mechanism, i.e., the formation of the compound nucleus. It also provides useful and practical information for theoretical predictions of the synthesis of SHE with various combinations of targets and incident ions.

\section{Acknowlegements}

The present work has been supported by JSPS grant No.18540268 and in part by JSPS Core-to-Core Program for Exotic Femto Systems. One of the authors (C.S.) thanks the supports from Natural Science Foundation of China and from Natural Science Foundation of Zhejiang Province under the grant Nos. 10675046 and Y605476, respectively. The authors also acknowledge supports and hospitality by RCNP Osaka Univ., GANIL, Huzhou Teachers College, and IPT, CE-Saclay, which enable us to continue the collaboration.

\section{References}

1. C. Shen, G. Kosenko, and Y. Abe, Phys. Rev. C66 (2002) 061602(R).

Y. Abe and B. Bouriquet, Acta Phys. Pol. B34 (2003) 1927-1945.

Y. Abe, et al., Prog. Theor. Phys. Suppl. No. 146(2002) 104-109.

W.J. Swiatecki, J. Siwek-Wilczynska and J. Wilczynski, Acta Phys. Pol. B34 (2003) 2049 and Phys. Rev. C71 (2005) 014602.

2. B. Bouriquet, G. Kosenko, and Y. Abe, Eur. Phys. J. A22 (2004)9.

Y. Abe, D. Boilley, G. Kosenko, and C. Shen, Acta Phys. Pol. B34 (2003)2091-2105.

3. B. Bouriquet, D. Boilley, and Y. Abe, Comp. Phys. Comm. 159 (2004) 1-18.

Y. Abe, et al., Intern. J. Modern Phys. E15 (2006) 491-501.

$\mathrm{Ph}$ D thesis of A. Marchix, GANIL(2007)

4. Y. Abe, Eur. Phys. J. A13 (2002)143-148.

Y. Abe, C. Shen, G. Kosenko, and D. Boilley, Phys. of At. Nucl. 66 (2003)1057-1064.

5. G. Kosenko, C. Shen, and Y. Abe, J. Nucl. Radiochem. Sci. 3 (2002)19-22.

6. Y. Abe, D. Boilley, B. Giraud, and T. Wada, Phys. Rev. E61 (2000) 1125-1133.

7. K. -H. Schmidt and W. Morawek, Rep. Prog. Phys. 54, (1991) 949.

8. D. Boilley, Y. Abe, and J. Bao, Eur. Phys. J. A18 (2003) 627-631.

9. A. Iwamoto et al., Prog. Theor. Phys. 55 (1976) 115. K. Sato et al., Z. Phys. A290 (1979)145.

10. W.J. Swiatecki, Phys. Scripta 24 (1981) 113. G.G. Adamian et al., Nucl. Phys. A619 (1997) 241. T. Wada, A. Fukushima, and M. Ohta, Proc. EXON2004, (World Sci.Inc.) p.255.

11. Y. Abe, S. Ayik, P.-G. Reinhard, and E. Suraud, Phys. Reports C275 (1996), Nos. 2 and 3.

12. M. v. Smoluchowski, Physik. Zeitschr. 17 (1916) 585. S. Chandrasekhar, Rev. Mod. Phys. 15 (1943) 1. 